\title{
¿QUÉ HAY QUE RENOVAR EN LOS TRABAJOS PRÁCTICOS?
}

\author{
GONZÁl,EZ EDUARDO, M. \\ Universidad Nacional de Córdoba, Argentina.
}

\section{SUMMARY}

The article starts presenting four innovative alternatives of practical work at the laboratory. After forestalling layouts intended to teach scientific procedures leaving out knowledge acquisition, we propound practical work, such as solving problems with capacity to grow building theoretical frames.

\section{INTRODUCCIÓN}

En la bibliografía y en los congresos sobre Didáctica de las Ciencias aparecen con frecuencia trabajos críticos y propuestas de renovación para la tarea del laboratoriso. La mayor parte đe ellos están đirigidos al nivel secundario, aunque también los hay abundantes para la universidad. Más allá de las lógicas diferencias, estos estudios tienen un rasgo en común: están destinađos a enfrentar de algún modo las limitaciones de los trabajos prácticos de «ilustración o verificación».

Las aportaciones críticas son amplias y diversas. Moreira (1980) afirma que «muchos estudiantes realizan un ex * perimento sin tener una idea clara de los que están haciendo; ellos no son capaces de identificar las cuestiones básicas, los conceptos y los fenómenos involucrados en el experimento, y, además, no ven la experimentación como un proceso de construcción del conocimiento». Hofstein y Lunetta (1982) señalan que las comparaciones entre distintos métodos de laboratorio y de éstos con otros medios de enseñanza más convencionales no han aportado diferencias significativas. Si bien enfatizan que se trata un medio "único» para la enseñanza de lá ciencia, reconocen la necesidad de nuevas investigaciones para establecer las relaciones entre la experiencia en el laboratorio y cl aprendizaje de los estudiantes.

Gil y Payá (1988) muestran en un estudio que «los trabajos prácticos de Física y Química habituales no familiarizan, ni siquiera mínimamente a los alumnos con la metodología científica», encontrándose en los mismos, «incluidos los textos y en la forma misma que el profesorado los plantea, una ausencia de aspectos fundamentales, como la cmisión de hipótesis o el mismo diseño a realizar». Señalan finalmente que «la indudable capacidad motivadora que los trabajos prácticos tienen a priori, se convierte en decepción después de realizarlos".

En cuanto a las tentativas renovadoras, los resultados son bastante contradictorios. En algunas experiencias cs posible constatar una combinación entre logros muy positivos, sobre todo en el aprendizaje de habilidades y en la motivación, y avances mucho más reducidos en los aspectos conceptuales y en los metodológicos (Buckmaster 1986, González y Frascino 1989).

¿Han sido éstos los resultados típicos que han producido Jas alternativas renovadoras? ¿Han modificado la investigación e innovación didáctica aquellas limitaciones señutadas en las prácticas tradicionales? ¿Siguen vigentes csos experimentos tipo recetas donde están ausentes aspectos fundamentales de la actividad de la ciencia? ¿Qué aspectos deben ser considerados para lograr un avance efectivo en el aprendizaje a través de los trabajos de laboratorio?

\section{CÓMO CARACTERIZAR IAAS PROPUESTAS RENOVADORAS}

Sin pretender abordar esta problemática en forma global, es posible hacer una contribución a la discusión de 
la misma a partir de analizar algunas de las orientaciones y de las aportaciones que reiteradamente se encuentran en esas alternativas renovadoras.

Para ello se partirá de uno de los objetivos más señizlados a la hora de fundamentar la tarea de laboratorio en la enscĩanza: acercar a la actividad y a los métodos de la ciencia. El hilo conductor estará dado entonces por las relaciones que puedan establecerse entre esos trabijos y el proceso de producción científica.

Desde este punto de vista podrían distinguirse cuatro grupos de propuestas. A continuación se describen esos grupos y se dan aigunas referencias de trabajos concretos (es posible ampliar las referencias utilizando las reseñas bibliográficas de Payá [1983], Pastor y López [1987], Furió [1989] y Payá [1990], que tienen una orientación similar a la presente).

1. Actividades a-teóricas. Serían aquéllas destinadas a mejorar las habilidades prácticas y los conocimientos técnicos, pero que están desligadas, parcial o totalmente, de un cuerpo teórico y de verdaderos problemas planteados en relación al mismo. Van desde la sugerencia de limitar el laboratorio a aquellos estudiantes que se proponen ser investigadores en la disciplina e iniciar la enseñanza de técnicas experimentales en forma «teórica», es decir, mediante descripciones verbales o mediante películas (Toothacker 1983); pasando por enfrentar a los alumrios con "problemas-desafío» (calcular el tamaño de un agujero o la velocidad de una rueda de bicicleta), donde se aplica la teoría de errores y se revisan relaciones entre magnitudes (Sebastiá 1985); hasta la realización de un curso sobre procesos científicos desconectado del desartollo de una materia (Braga 1987).

2. Desarrollo de recursos (humanos, materiales y didácticos). Se refiere a aquellas activiđades destinadas a la preparacion y el mejoramiento de los distintos elementos que sc integran en la práctica, pero que no determinan el contenido de la misma. Se incluyen aquí: las propuestas para introducir los equipos de bajo costo (Frascino y Gonzálę 1989); la sugerencia de dar una formación específica a los profesores para la enseñanza en el laboratorio (Tamir 1989); trabajos donde se ha establecido que una de las razones del rechazo de los estudiantes a laas prácticas de laboratorio o de la ineficacia del aprendizaje por estc medio se encuentra en una «sobrecarga teórica» (Johnstone 1990); criterios para mejorar la «rclación señal-ruido» en el laboratorio, es decir para una mejor estructuración de la actividad (Byrne 1990); o finalmente programas de investigación donde, entre otras cosas, se vuelca al laboratorio Ia utilización de lo cotidiano (Menezes 1990).

3. Aplicaciones nuevas o problemáticas. En este caso los cambios están referidos a una nueva manera de enfrentarse con los contenidos que se han dado en la teoría. Habría, por un lado, una enorme cantidad de trabajos sobre temas puntuales, en los que generalmente se ofrecen enfoques y experiencias originales; $y$, por el otro, planteos más globales donde se intentan transformar los trabajos prácticos para convertirlos en verdaderas situaciones problemáticas, similares a las de una investigación (Raghubir 1979, Arena 1989 y Dumon 1991). Estas orientaciones suelen estar acompañadas deI desarrolio de nuevos manuales e implican una formulación diferente de la actividad, como los «laboratorios semiestructurados en un modelo de investigación simplificado» (García de Sarmiento 1989).

\section{Pequeñas investigaciones dirigidas. A diferencia del} punto anterior, los problemas que se abordan no están destinados solamente a aplicar los conocimientos que ya se han dado, sino que se refieren incluso al desarrollo de los mismos. Se trata de tentativas de acercar los trabajos prácticos a una verdadera tarea de investigación, donde se reproduce el proceso de descubrimiento de una manera afín a la de la ciencia. Es decir, que sc vincula la tarea de laboratorio con la construcción del conocimiento, que pasa a ser entonces una «actividad coherente con el trabajo cicntífico» (Gil et al. 1980, Gené 1986, Gil y Payá 1988).

\section{NOHAY EXPERIMENTOS SIN CONTENIDOS}

El desarrollo de actividades originales y creativas, aun desvinculadas de contenidos teóricos, puede ser útil para el aprendizaje de habilidades y técnicas. Es más, en cicrtos contextos (ausencia de una experiencia anterior, espectacularidad, aspectos novedosos) estas tareas sucien ser una fuente de motivación.

Por otra parte, hay que considerar que la observación de ciertos fenómenos en una versión inductivista acerca parcialmente a situaciones reales de una investigación; situaciones, en las que el problema no está claro, etapas de tanteo, ctc. Es posible que a través de estas actividades los estudiantes logren una familiarización con algunas de las tareas del laboratorio y que se predispongan para abordajes más amplios.

Ello no puede en modo alguno suplir o reemplazar la enseñanza de otros aspectos fundamentales de lo que se llama el método científico. Si se limitara la tarea de laboratorio a estos ejercicios empiristas podría inducirse una falsa imagen sobre Io que es la tarea de la ciencia, que no emplea técnicas y métodos en abstracto sino con relación a contenidos y problemas concrctos (Hodson 1988). Como se ha señalado, la obtención de evidencia experimental sólo cobra sentido con relación a la hipótesis a contrastar y a los diseños concebidos a tal efecto (Gil 1983).

Por otra parte, cabría considerar si es ventajoso o perjudicial para la formación de los estudiantes motivarlos con trabajos desligados de un contenido conceptual. Ya en etapas muy tempranas se ha mostrado que la adquisición de habilidades y destrezas y el desarrollo del interés se relacionan con actividades problemáticas que ponen en cuestión algún conocimicnto (Blueford 1989).

Esto no significa emitir un juicio de valor sobre la utilidad parcial que pueden tener propuestas como Ias señaladas, tanto como generadoras de motivación $y$ 
experiencia, como en el aprendizaje de técnicas y rutinas de trabajo. Incluso se las podría reconvertir para aprovechar desde otro marco algunas de sus aportaciones; ya sea integrándolas a orientaciones de trabajos prácticos que no eludan lo conceptual, ya sea formando parte de cursos de taller.

La conveniencia o no de esos cursos está fuera del marco de esta discusión. I o que se considera aquí como un error es la creencia de que el aprendizaje de técnicas debe preceder o realizarse al margen del iratamiento de verdaderos problemas y, peor aún, que debe reemplazarlo. En última instancia, el enor consiste en seguir sosteniendo puntos de vista que han sido ampliamente rechazados en la filosolía de la ciencia: la existencia de un método general de la ciencia independiente de los contenidos (Gil 1983 y Hodson 1988).

\section{MODIFICAR EL CONTEXTO Y LA CON- CEPCION DIDACTICA}

Las proposiciones de mejorar los recursos y su utilizacion apuntan a resolver diversos problemas concretos que se presentan a la hora de realizar la actividad de laboratorio. Se trata de aspectos parciales, de contexto, pero que tienen mucha importancia. Estas cuestiones adquicren a veces un tono dramático (escuelas carentes de todo equipamiento, profesores sin formación específica, etc.) y pueden ser determinantes para hacer inviable cualquier proyecto de mejoras.

Estas orientaciones acercan las tentativas de cambio a un trabajo más implicado, que parte de considerar las limitaciones que impone la realidad y que se propone abordarlas a través de un esfuerzo sistemático. Al mismo tiempo se generan canales que facilitan la participación y sensibilización de los docentes y la de los propios estudiantes.

De esta manera se consiguen dos ventajas adicionales; por un lado se contribuye a mejorar el «clima del centro»; por el otro se facilita un mayor grado de participación de los estudiantes en el trabajo práctico del laboratorio. Sc trata de aspectos de una gran importancia en el desarrollo de actitudes positivas, tanto hacia la disciplina como hacia cl laboratorio (Rivas 1986 y Okebukota 1986).

Sin embargo, se corre cl riesgo de que, si el problema que dio origen a la propuesta no está suficientemente elaborado e inserto en una concepción más global, estas tareas lleven a confusión y no permitan superar la perspectiva tradicional. Así, por ejemplo, la introducción de equipos de bajo costo, que en ciertos casos constituye un primer paso saludable y motivador, puede al mismo tiempo empalmar con una óptica simplista, que apela más bien a la receta, y que intenta producir transformaciones por la simple actividad (González y Frascino 1989). De la misma manera, los criterios de reducir la «sobrecarga teórica», mejorar la «relación señal-ruido» e ideas de ese tipo, que parecen interesantes para mejorar la programación de los trabajos prácticos, pueden conducir también a la minimización de los contenidos y a un planteo simplifícador.

Por distintos caminos se terminaría cayendo en una reiteración de los defectos criticados anteriormente: trabajos prácticos rutinarios, sin contenidos o carentes de aspectos sustanciales de la tarea de la ciencia. Esto podría a su vez originar un desgaste muy lamentable, que afectaría justamente a aquellos profesores que más cmpeño han puesto en la tarea.

De modo que, por un lado, el desarrollo de esos recursos es una tarea muy importante para la estructuración del contex to $y$, por el otro, esa misma actividad puede hacer perder la perspectiva sobre lo que se quiere y se debe hacer con los trabajos prácticos. Se trata, por lo tanto, de establecer correctamente la relacion entre los objetivos generales y los medios necesarios para lograrlos.

Así, las estrategias de Tamir (1989) para perfeccionar a los profesores cncargados de impartir la enseñanza experimental, o las sugerencias de Barbara (1989) de enseñar a los estudiantes a redactar informes, que se integran en un proyecto de «laboratorio por investigación", son ejemplos claros de tentativas de vincular una modifícación del contexto a una perspectiva globalizadora de accrcar la enseñanza en el laboratorio a la actividad de la ciencia.

Más en perspectiva, se trata de un problema que supera la cuestión de los trabajos prácticos y tiene que ver, entre otras cosas, con las concepciones de los profesores respecto de los problemas docentes. Como han señalado Furió y Gil (1989) de nada servirán las propucstas renovadoras que no se integren en esas concepciones 0 que no intenten modificarlas.

\section{APLICACIONES, PROBLEMAS E ILUSTRA- CIÓN DE CONCEPTOS}

Las propuestas que introducen nuevas aplicaciones o que proponen situaciones problemáticas utilizando clementos conceptuales de la materia contienen una ventaja clara sobre las tentativas a-teóricas. Los caminos a través de los cuales se intenta romper con las rutinas tradicionales y facilitar que el estudiante adquiera «soltura experimental" ya no eluden la relación necesaria entre el experimento y la estructura conceptual. Es más, la realización de la tarea requiere en muchos casos de un esfuerzo imaginativo y de una profundización de los contenidos.

La idea de enfrentar situaciones problemáticas, planteadas a partir de marcos teóricos ya establecidos, es un criterio muy flexible, que permite conectar con astuntos de la vida diaria y de la tecnología. En la tarea de resolver esos problemas los estudiantes son colocados en una situación que es habitual en una investigación científica, que generalmente comienza con el planteamiento de un problema (Gil et al. 1980). 
Algunos de estos trabajos constituyen verdaderos ejercicios teórico-prácticos de aplicación, que no pueđen ser resueltos sin combinar un aspecto cualitativo de «enredarse con el fenómeno" (Driver 1986), con las herramientas y el rigor del análisis cuantitativo. Incluso pueden llevar a que los trabajos prácticos contengan el planteamiento del problema, la emisión de hipótesis y el discño deI experimento, algo que se viene reclamando desde los años sesenta, pero que está muy lejos de haberse conseguido en la práctica (Fuhrman et al. 1982, Gil 1983).

Los resultados de estas actividades son muy alentadores, tanto en la adquisición de habilidades, como en la motivación o en el desarrollo de actitudes positivas hacia la tarea del laboratorio (Penick y Yager 1986, Gomes y Penna 1988, Dumon 1991). Parece que esta perspectiva facilita un canal válido para đesarrollar la iniciativa y la creatividad de los estudiantes.

Las conclusiones son más limitadas en cuanto a la «ilustración de conceptos o a la aportación de nuevas ideas" (Arena 1989). Esta cuestión sugiere la necesidad de discutir las relaciones entre la situación-problema, la manera de abordarlo y los conceptos que están involucrados.

Si bien estas tareas no están planteadas en relación a la elaboración de contenidos, elío no elimina la posibilidad de que las mismas aporten matices o completen el significado de los conceptos en juego. En el caso de que la aplicación novedosa o la situación-problema carezca de estos aspectos, la tarea aparecerá muy acotada y acercándose a la «verificación» tradicional. Si por el contrario se diluyera la relación entre el problema y la tase conceptual (búsquedas observacionales, hacer eje en los métodos y no en los contenidos), la tarea se acercará al inductivismo. De ambas maneras el trabajo práctico perdería parte de su potencial educativo.

En definitiva, el aspecto creativo que aporta este tipo de prácticas está limitado por el carácter ilustrativo quei le impone un marco teórico ya acabado. Ello remite a su vez al mantenimiento de las clases teóricas tradicionales, necesariamente dogmáticas, de trasmisión de conocimientos ya elaborados (Gíl et al. 1980).

\section{LABORATORIO Y DESARROLLO DEL MARCO TEORICO}

La discusión de las orientaciones señaladas anteriormente nos plantea la necesidad de analizar en profundidad las relaciones entre la tarea que se aborda en les actividades prácticas y el marco teórico en el que se va a trabajar. En este sentido, la orientación de vincular los trabajos prácticos de laboratorio con la construcción del cuerpo de conocimientos puede ser vista como un planteo que contiene ese aspecto desarrollado en su totalidad.

Se ha señalado que uno de los requisitos para que el trabajo de laboratorio sea convincente es que la activi. dad de los estudiantes se acerque al comportamiento de un auténtico investigador, que puede contrastar sus conjeturas y clegir entre varios caminos (Solomon 1988).

Pero la tarea de un investigador suele estar dirigida tanto a desarrollar sus hipótesis a partir de una teoría bien establecida, como a desarrollar esta última.

Es posible concebir del mismo modo el proceso de enseñanza-aprendizaje y consecuentemente el sentido del laboratorio. No se trata sólo de un aprendizaje de métodos o de una ilustración de la teoría, ni se trata exclusivamente de aplicar esa teoría a la resolución de problemas. Se trata de dar un significado en el aprendizaje al hecho de que la ciencia es una actividad teóricoexperimental.

La idea subyacente a estas orientaciones es que puede introducirse algún paralelismo entre el proceso de enseñanza-aprendizaje y la actividad de investigación científica (Gil 1983). Este tipo de laboratorio requiere integrar técricas, ilustraciones paradigmáticas, tratamiento de los datos, resolución de problemas, pequeñas investigaciones, desarrollo de conceptos, diseño de experimentos, discusión colectiva de resultados, etc.; todo ello a través de un proceso integral orientado por el docente. De este modo, la tarea educativa queda planteada de una manera análoga a lo que es una investigación dirigida (Gil, Carrascosa, Furió et al. 1988).

Una orientación de este tipo permite superar tanto los planteos inductivistas o descontextuados que suelen aparecer en aproximaciones intuitivas, como los esquemas de trasmisión-verificación que se heredan de los modelos tradicionales. Al mismo tiempo permite integrar de manera coherente las aportaciones positivas que siempre están presentes en dichas tentativas.

\section{CONCLUSIÓN}

Esta comparación sugiere el estado de desarrollo de las distintas concepciones y experiencias didácticas para mejorar la tarea de laboratorio. Parece necesario continuar avanzando en la perspectiva de vincular el laboratorio con to que es la actividad de investigación en la ciencia.

Aunque los resultados aIcanzados en este tipo de propuestas son muy alentadores, sobre todo en la escuela secundaria, no puede decirse que las mismas se hayan generalizado o que se hayan explorado en profundidad sus posibilidades. Se trata de un proceso en desarrollo que requiere de nuevas investigaciones.

En particular, parece interesante llevar estos estudios al nivel universitario, donde todavía no se han reflejado mayormente los resultados de la investigación dídáctica. Más todavía cuando, a diferencia de la escuela secundaria, en la universidad sí que se realizan abundantes trabajos de laboratorio.

En todo caso, está claro que se trata de una actividad muy implicada, que requicre de un trabajo previo que es 
mayor que el de las orientaciones tradicionales, que exige una preparación adecuada de los docentes (Gif et al. 1990) -para dar información en el momento adecuado, para reorientar, etc.- y que va a modificar tanto la relación docente-alumno (Gil 1983), como la evaluación (Giddings y Hofstein 1980).

Ello plantea, a su vez, la necesidad de replantear en profundidad la actividad docente. Supone tomar conciencia de que enseñar no es tan fácil que baste algo de sentido

\section{REFERENCIAS BIBLJOGRÁFICAS}

ARENA, L. E., DE LONGHI, A. I. y BRUNETTI, A., 1989. El laboratorio de física: un cambio metodológico, REF., 6, pp. 264-275. (Bariloche: Argentina).

BARBARA, Y., 1989. Merging Scientific writing with the investigative laboratory, Journal of College Science Teaching, pp. 94-95.

BI,UEFORD, J. R., 1989. A guide to hands-on science, Science and Children, pp. 20-21.

BRAGA, I. L.., 1987. ¿Ingresan los alumnos en la Universidad con un adecuado desartollo de los niveles de razonamiento?, Enseñanza de las Ciencias, 5 (1), pp. 16-21.

BUCKMASTER, HX. A., I986. Evaluating the effectiveness of an elementary undergraduate laboratory, American Journal of Physics, 54 (8), pp. 702-704.

BYRNE, M. S, 1990. More effective practical work, Education in Chemistry, pp. 12-13.

DRIVER, R., 1986. Psicología cognoscitiva y esquemas conceptuales de los alumnos, Enseñanza de las Ciencias, 4 (1), pp. 3-15.

DLMON, A., 1992. Former les Étudiants a la demarche experimentale: utopic ou question depassée?, Enseñanza de las Ciencias, $10(1)$.

FRASCINO, A. y GONZÁLEZ, E, 1989. Propuesta para la utilización de los FBC en la enseñanza de la física en el nivel medio. (Universidad Nacional de Córdoba: Argentina).

FUHRMAN, M., LUNFTYA, M. y NOVICK, S., 1982. Do secondary school laboratory texts reflect the goals of the new science curticula?, Joumal of Chemical Education, 59, pp. 563-565.

FURIÓ, C., 1989. Selección bibliográfica sobre evaluación de prácticas de laboratorio, Enseñanza de las Ciencias, 7 (2), p. 187.

FURIÓ MAS, C.J. y GIL PÉREZZ, D., 1989. La didáctica de las ciencias en la formación inicial del profesorado: una orientación y un programa teóricamente fundamentados, Enseñanza de las Ciencias, 7 (3), pp. 257-265.

GENÉ, A., 1986. Transformació dels treballs pràctics de Biologia. Una proposta teòricament fonamentada. 'Tesis Doctoral, Universitat de Barcelona. común, experiencia y conocimiento de la materia y supone también adquirir conocimientos teórico-prácticos sobre la enseñanza-aprendizaje de las materias científicas (Gil et al. 1990).

Se trata, sin duda, de la orientación más ardua y compleja, pero también de la más prometedora. Es también una posibilidad para que los esfuerzos innovadores de los docentes y las expectativas de los estudiantes no terminen en nuevas frustraciones.

GIDDINGS, G, y HOFSTFIN, A., 1980. Trends in the assessment of laboratory performance in high school science instruction. The Australian Science Teachers Journal, 26 (3), pp. 57-64.

GII, D., CALATAYUD, L., GINER, F, ORTIS, E., SFRO, E. y SEVILLA, C., 1980. Trabajos Prácticos de Físicu como pequeñas investigaciones. Instituto de Ctencias de la Lducacion, Universidad de Valencia.

GIL, D., 1983. Tres paradigmas básicos en la enseñanza de las ciencias, Enseñanza de las Ciencias, pp. 26-33.

GIL, D., CARRASCOSA, J., FURIÓ, C. et al., 1988. La Construcción de las Ciencias Físico-Químicas, Programasguía de trabajo y comentarios para el profesor. (NAU 1libres; València).

GIL, D. y PAYA, J., 1988. Los trabajos prácticos de Fisica y Química y la metodología científica, Revista de Enseñanza de la Física, 2 (2), pp.73-79

GIL, D., BELENDEZ, $\Lambda$, MAR'TIN, A. Y MARTINYYZ TORREGROSA, J., 1990. La formación del profesorado uni. versitario de materias científicas: contra algunas ideas - y comportamientos de "sentido común». It Jornadas Nacionales de Didáctica Universitaria: Alicante.

GONZÁLEZ, E. y IRASCINO, A., 1989. Un análisis de las posibilidades de transferencia de los $\mathrm{BBC}$, Trabajos de Educación en Ciencias, 2.

GOMES, P. R. S. y PLNNA, T. J. P., 1988. Proposta de uma disciplina com enfoque na metodologia da fisica experinental, Revista de Ensino de Física, 10, pp. 34-42.

HODSON, D., 1988. Toward a philosophically more valid science curriculum, Science Education, 72 (1), pp. 19.40 .

HOFSTEIN, A. Y LUNETTA, V. N., 1982. The role of the laboratory in science teaching; neglected aspects of research, Review of Educational Research, S2 (2), pp. 201-217.

JOHNSTONE, A. H. y I.ETTON, K. M., 1990. Investigating undergraduate laboratory work, Education in Chemistry, $9+11$.

MFNEZLSS, C., KAWAMURA, M. R., HOSOUME, Y. y ZANETIC, J., 1990 . Renovaçao de contédos e formascao de professores. Actas do IIY Encontro de Pesquisa em Ensino do Fisica, Porto Alegre, pp. 64-68. 
MOREIRA, M. A., 1980. A non-traditional approach to the evaluation of laboratory instruction in general physics, Furopean Journal in Science Education, 21, p. 441.

OKEBUKOLA, P. A., 1986. An investigation of some factors affecting students ${ }^{\dagger}$ attitudes toward laboratory Chemistry, Journal of Chemical Education, 63 (6), pp. 531-532.

PASTOR, J. M. y LÓPEZ, M. 1987. La investigación didáctica sobre los trabajos prácticos: una selección bibliográfica (1980-1985), Enseñanza de las Ciencias, 5 (3), p. 262.

PAYA, J., 1983. Los trabajos prácticos de física y de química: una selección bibliográfica, Enseñanza de las Ciencias, I(2), pp. 131-132.

PAYÁ, J., 1990. Los trabajos prácticos de física y química: una revisión bibliográfica, Enseñanza de las Ciencias, 8 (2), pp. $181-185$.

PENICK, J. E. y YAGER, R. E., 1986. Trends in science education: some observations of exemplary programmes in the United States, European Jownal in Science Education, 8 (1), pp. 1-8.

RAGHURIR, K. P., 1979. The laboratory-investigative approach to science instruction, Journal of Research in Science Teaching, 16 (1), pp. 13-17.

RIVAS NAVARRO, M, 1986. Factores de eficacia escolar: una línea de investigación didáctica, Bordón, 2 (64), Tomo XXXVIII, pp. 693-707.

SEBASTIA, J.M., 1985. Las clases de laboratorio de Física: Una propuesta para su mejora, Enseñanza de las Ciencias, 3 (1), pp. 42-45.

SOLOMON, J., 1988. Learning trough experiment, Studies in Scientce Education, 15, pp. 103-108.

TAMIR, P., 1989. Training teachers to teach effectively in the laboratory, Science teacher education, 73 (1), pp. 59-69.

TOOTHACKER, W. S., 1983. A critical look at introductory laboratory instruction, American Journal of Physics, 51 (6). 